\title{
Interaction fluide structure pour des faisceaux de tubes : Méthodes d'homogénéisation, analyse physique
}

\author{
DANiel Broc ${ }^{1, a}$ et JeAn-FranÇois Sigrist ${ }^{2}$ \\ 1 CEA Saclay DEN/DM2S/SEMT/EMSI, 91191 Gif-sur-Yvette Cedex, France \\ 2 DCNS Propulsion, Service Technique et Scientifique, 44620 La Montagne, France
}

Reçu le 30 septembre 2008, accepté le 9 février 2009

\begin{abstract}
Résumé - Les interactions entre les mouvements d'une structure et d'un fluide sont à l'origine de phénomènes dynamiques (vibratoires ou accidentels) dont la maîtrise est indispensable pour résoudre un bon nombre de problèmes pratiques posés lors de la conception et de l'exploitation d'installations industrielles. On rencontre fréquemment dans l'industrie, en particulier dans l'industrie nucléaire, des faisceaux de tubes immergés dans un fluide. Les principales conséquences de la présence du fluide sont des effets « inertiels », amenant une baisse des fréquences propres de vibration et des effets « dissipatifs », amenant une augmentation de l'amortissement. L'article présente d'abord un état des méthodes actuelles pour la simulation du comportement dynamique de faisceaux de tubes, avec des exemples d'application. Ces méthodes sont basées sur l'homogénéisation des équations d'Euler d'un écoulement de fluide parfait. Il est possible ainsi de rendre compte des effets inertiels et aussi, dans une certaine, des effets dissipatifs. La conclusion indique des voies de recherche pour une prise en compte plus complète de la présence du fluide, en particulier des effets dissipatifs.
\end{abstract}

Mots clés : Interaction fluide structure / faisceaux de tube

\begin{abstract}
Fluid-structure interaction in tube bundles: homogenisation methods, physical analysis. It is well known that the movements of a structure may be strongly influenced by fluid. This topic, called "Fluid Structure Interaction" is important in many industrial applications. Tube bundles immersed in fluid are found in many cases, especially in nuclear industry: (core reactors, steam generators,...). The fluid leads to "inertial effects" (with a decrease of the vibration frequencies) and "dissipative effects" (with higher damping). The paper first presents the methods used for the simulation of the dynamic behaviour of tube bundles immersed in a fluid, with industrial examples. The methods used are based on the Euler equations for the fluid (perfect fluid), which allow to take into account the inertial effects. It is possible to take into account dissipative effects also, by using a Rayleigh damping. The conclusion focuses on improvements of the methods, in order to take into account with more accuracy the influence of the fluid, mainly the dissipative effects, which may be very important, especially in the case of a global fluid flow.
\end{abstract}

Key words: Fluid structure interaction / tubes bundles

\section{Introduction}

L'interaction fluide structure (ou IFS) est l'étude de l'influence d'un fluide sur le comportement dynamique de structures. C'est un vaste domaine d'étude, qui inclut de nombreuses et différentes configurations industrielles [1-3].

\footnotetext{
a Auteur pour correspondance : dbroc@cea.fr
}

L'analyse de ce type de problème peut être complexe, tant du point de vue de l'interprétation physique que du point de vue du traitement numérique des équations correspondantes. Dans le cas le plus général, il est nécessaire, pour construire un modèle numérique de comportement, de réaliser un couplage entre les équations régissant le comportement de la structure et les équations générales de Navier-Stokes pour le fluide. En fonction des conditions, des simplifications peuvent être faites. 


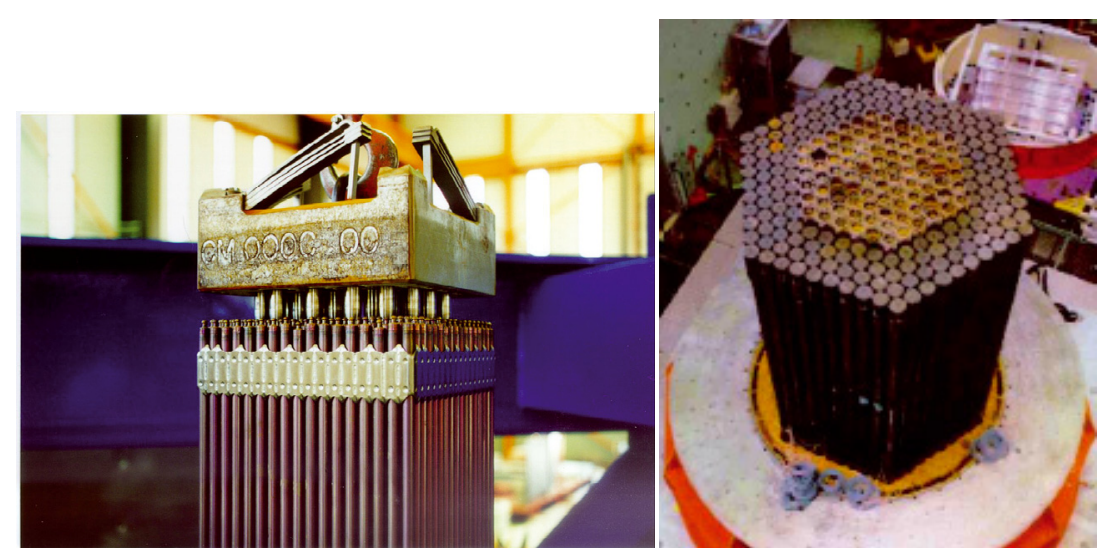

Fig. 1. Assemblage combustible REP et cœur RNR.

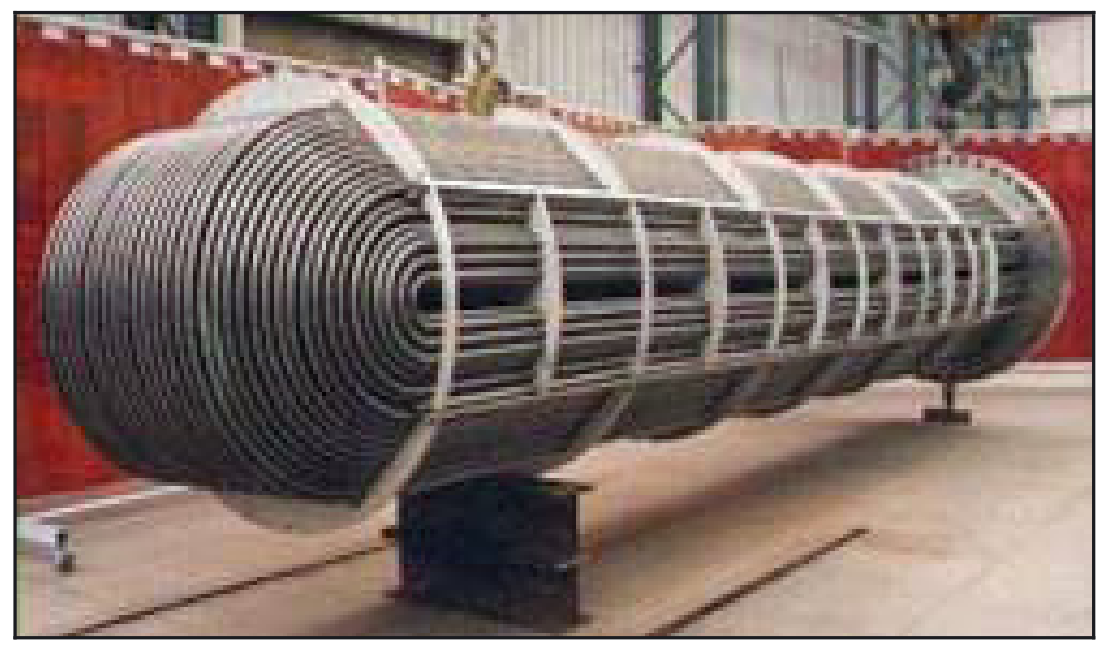

Fig. 2. Générateur de vapeur REP (en position couchée).

Un cas particulièrement important d'IFS, rencontré notamment dans l'industrie nucléaire, est celui de la vibration de faisceaux de tubes immergés dans un fluide (voir Figs. 1 et 2) :

- dans les cours de réacteur REP;

- dans les cours de réacteur RNR-Na;

- dans les cours de réacteurs embarqués ;

- dans les générateurs de vapeur.

Ces différents exemples de faisceaux de tubes immergés dans un fluide se différencient par les dimensions des tubes, leur nombre, leur confinement, la présence ou non d'un écoulement de fluide, à l'intérieur ou à l'extérieur des tubes, la possibilité d'impacts entre les tubes, etc. Les enjeux industriels peuvent être :

- le comportement en fonctionnement normal, avec des phénomènes de vibrations sous écoulement;

- la réponse à une sollicitation dynamique externe (séisme, impact, ou autre), où l'on cherche, suivant les cas, à contrôler les déplacements des tubes (déplacements absolus ou relatifs) ou les impacts entre les tubes.

\section{Méthodes de simulation du comportement dynamique de faisceaux de tubes}

\section{1 Équations générales}

Structure : les tubes sont considérés comme des systèmes à 2 degrés de liberté (en $x$ et en $y$ ), de masse $m$, de pulsation propre $\omega_{\mathrm{s}}$. Les équations régissant leur comportement sont :

$$
m \ddot{X}_{\mathrm{s}}+m \omega_{\mathrm{s}}^{2} X_{\mathrm{s}}=F
$$

oú $\ddot{X}_{\mathrm{s}}$ et $X_{\mathrm{s}}$ sont l'accélération et le déplacement du tube, $F$ est la force appliquée par le fluide.

Fluide : les équations générales régissant le comportement d'un fluide incompressible sont les équations de Navier Stokes :

$$
\rho \frac{\mathrm{d} V}{\mathrm{~d} t}=-\vec{\nabla} P+\mu \Delta V
$$

( $V$ est la vitesse du fluide et $\mu$ la viscosité) et l'équation de continuité :

$$
\operatorname{div} \nu_{F}=0 . \quad P . V=0
$$


Interface entre le fluide et la structure : dans le cas général (équations de Navier Stokes) la continuité des vitesses est assurée à l'interface $\Gamma_{\mathrm{s}}$ entre le fluide et le solide. Lorsque les équations d'Euler sont utilisées, les efforts appliqués par le fluide sur la structure sont limités aux termes de pression :

$$
F=\int_{\Gamma_{\mathrm{s}}} P \vec{n} \mathrm{~d} \Gamma .
$$

La continuité à l'interface pour le fluide et pour la structure se traduit par des expressions du type (exemple pour l'accélération) : $\ddot{X}_{\mathrm{S}} \vec{n}=V \vec{n}$.

\subsection{Simplification des équations décrivant le fluide}

Une des conséquences de la présence d'un fluide est la baisse des fréquences de vibration des structures. Les modèles d'écoulement de fluide parfait permettent de rendre compte de ces phénomènes.

Sous l'hypothèse de petits déplacements des structures la notion de « masse ajoutée » apparaît naturellement; le fluide est mis en mouvement par la structure : l'énergie cinétique est plus importante, et, pour une raideur donnée, la fréquence est plus faible.

Les équations d'Euler sont alors utilisées :

$$
\rho \frac{\partial V_{\mathrm{F}}}{\partial t}=-\vec{\nabla} P
$$

et l'équation de continuité $: \operatorname{div} V_{\mathrm{F}}=0$. Cette équation ne rend compte, en principe, que des effets inertiels de la présence du fluide. Les effets dissipatifs peuvent cependant être pris en compte sous la forme d'un amortissement forfaitaire de type « amortissement de Rayleigh ».

\subsection{Méthodes d'homogénéisation}

La géométrie d'un faisceau de tubes est complexe, bien que répétitive. La résolution des équations sur un faisceau décrit explicitement est lourde, voire impossible. Un important travail d'analyse a été mené dans les dernières années sur ce sujet [4-8]. Des méthodes d'homogénéisation ont été développées, qui, en s'appuyant sur la répétitivité du système, réduisent une cellule élémentaire (un tube) à un système à deux degrés de liberté : un degré pour le fluide et un pour la structure (voir Fig. 3) [9-14]. L'accélération du solide $\ddot{X}_{\mathrm{S}}$ est le degré de liberté pour le solide. L'accélération du fluide est le deuxième degré de liberté : on considère $\ddot{X}_{\mathrm{F}}$, flux échangé avec les cellules adjacentes. Les champs de vitesse (ou d'accélération) dans une cellule sont une combinaison linéaire des deux champs présentés sur la figure 3 :

- dans la partie gauche de la figure, l'accélération du fluide est égale à 1 , et celle du tube est égale à 0 ;

- dans la partie droite de la figure, l'accélération du fluide est égale à 0 , et celle du tube égale à 1 . L'accélération moyenne du fluide est négative, mais le flux échangé avec les cellules voisines est nul.

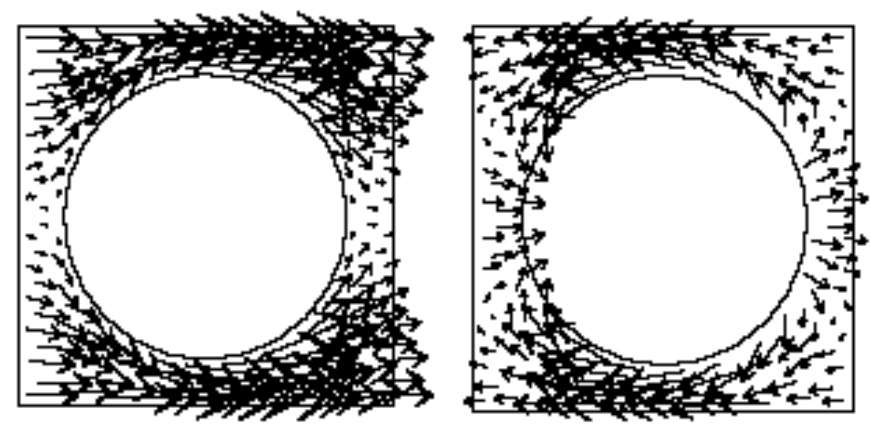

Fig. 3. Champs de vitesses dans une cellule élémentaire.

Les efforts appliqués par le fluide sur le tube sont :

$$
F=-m_{\mathrm{a}} \ddot{X}_{\mathrm{S}}+\left(m_{\mathrm{a}}+m_{\mathrm{d}}\right) \ddot{X}_{\mathrm{L}},
$$

où $m_{\mathrm{a}}$ est la «masse ajoutée » et $m_{\mathrm{d}}$ la masse d'eau déplacée par le tube.

À partir de l'expression des efforts échangés entre le fluide et la structure, on obtient l'équation suivante pour le fluide :

$$
\rho \ddot{X}_{\mathrm{F}}=-(1-J) \vec{\nabla} P+\rho J \ddot{X}_{\mathrm{s}}
$$

$P$ est la pression du fluide. Le coefficient $J$ est lié à la « masse ajoutée ». Cette équation est une modification de l'équation de fluide parfait (équation d'Euler) dans laquelle le coefficient $J$ réalise le couplage avec la structure.

On peut noter que, si les accélérations du fluide et de la structure sont identiques $\ddot{X}_{\mathrm{F}}=\ddot{X}_{\mathrm{s}}$, l'équation proposée se ramène à l'équation générale pour un écoulement de fluide parfait, $\rho \ddot{X}_{\mathrm{F}}=-\vec{\nabla} P$.

Cette méthode peut être généralisée en 3 dimensions. Elle a été intégrée dans CAST3M, code de calcul développé au CEA Saclay [15] en adaptant un formalisme général développé pour l'interaction fluide structure dans le cas d'un fluide parfait globalement immobile [16]. Les éléments homogénéisés ne sont que des éléments parmi d'autres, avec des degrés de liberté solide (déplacements) et des degrés de liberté fluide (pression).

\subsubsection{Intérêt et limites de la méthode d'homogénéisation mise en œuvre}

La méthode d'homogénéisation présentée est destinée à être appliquée aux vibrations de faisceaux de tubes immergés dans un fluide. Elle présente deux limites principales :

- les équations d'Euler ne sont théoriquement valables que si le fluide est globalement au repos et si les niveaux de déplacements sont faibles (très inférieurs à la dimension des tubes du faisceau). Dans le cas général, c'est-à-dire si les conditions précédentes ne sont pas réunies (écoulement du fluide ou déplacements importants), le fluide amène des effets dits « dissipatifs», qui se traduisent par un amortissement. Il est toutefois possible de rendre compte de cet amortissement, tout en conservant la méthode 

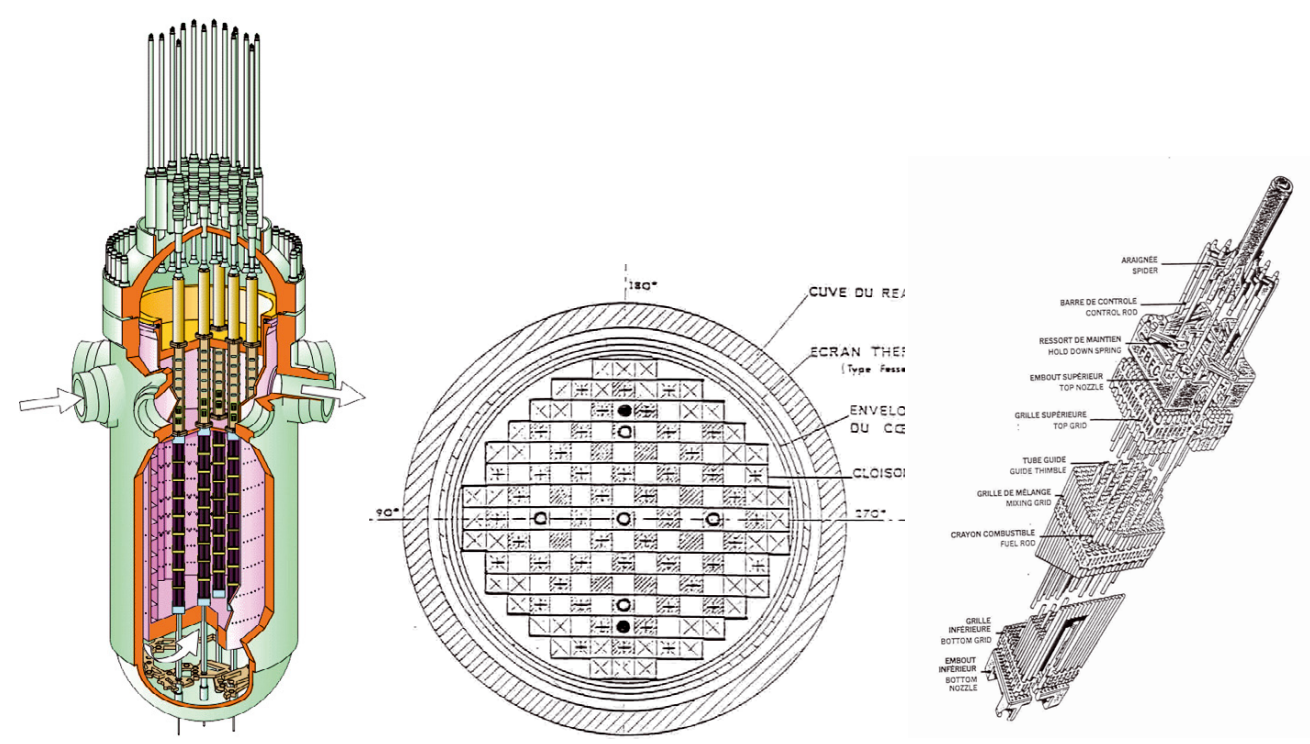

Fig. 4. Présentation d'un cœur de réacteur REP, vue générale, vue de dessus, assemblage.

d'homogénéisation présentée ici, en utilisant un amortissement forfaitaire (amortissement de Rayleigh). Les limites de ce type de prise en compte de l'amortissement restent à évaluer.

- Le fait de considérer que la cellule est un système à 2 degrés de liberté correspond à considérer que les accélérations du fluide et du solide varient peu entre une cellule et la cellule voisine. De fortes différences des accélérations de tubes voisins peut modifier le comportement du faisceau.

\section{Exemples}

Des illustrations sont présentées sur le comportement vibratoire de faisceaux de tubes pour différentes configurations :

- les réacteurs REP;

- un faisceau de tubes $10 \times 10$.

Les simulations présentées sont, suivant les cas, réalisées :

- par calcul « direct »(sans homogénéisation);

- en utilisant les méthodes d'homogénéisation;

- par les deux méthodes, dans un but de validation.

\subsection{Comportement dynamique et sismique des cœurs REP}

Les cœurs des réacteurs à eau pressurisée (REP) sont constitués de 157 assemblages. Ces assemblages sont de poutres carrées constituées de $17 \times 17=289$ tubes (Fig. 4).

La méthode d'homogénéisation décrite a été utilisée pour déterminer les $157 \times 2=314$ modes de vibration d'un cœur, en $2 \mathrm{D}$. Un élément homogénéisé correspond à un assemblage, avec 2 degrés de liberté pour le mouvement de l'assemblage ( $x$ et $y$ ) et une inconnue en pression à chaque coin du carré. La figure 5 présente des exemples de ces modes. La baisse de fréquence due à la présence du fluide est d'environ $10 \%$ à $15 \%$ suivant les cas.

Le premier mode présenté est un des 192 modes pour lesquels la fréquence est minimale. Ces modes correspondent à un mouvement de fluide global nul (pas d'échanges de fluide entre les cellules). Dans ces conditions, l'interaction fluide structure est maximale, d'où les faibles fréquences (15\% de moins qu'en air). L'allure générale de ce mode semble complexe. Il faut considérer que ce mode est la combinaison linéaire de 192 modes de même fréquence, d'allure plus organisée (comme le deuxième mode présenté sur la Fig. 5).

Les cing autres modes présentés sont plus « organisés », sous forme de cellules. Les accélérations du fluide ne sont plus nulles, mais sont proportionnelles aux accélérations des assemblages : l'interaction fluide structure est plus faible et les fréquences sont d'autant plus élevées que les cellules sont amples et peu nombreuses. Ces fréquences restent, dans tous les cas, inférieures aux fréquences en air.

La figure 6 présente deux schémas des types de mouvement des assemblages. Le premier schéma présente un mouvement d'ensemble des assemblages. C'est le seul type de mouvement qui prendrait place s'il n'y avait pas d'impacts entre les assemblages. Ce mouvement s'effectue avec la fréquence minimale : les mouvements globaux du fluide, il n'y a pas de transfert de fluide d'une cellule à une autre. Le deuxième schéma montre des mouvements relatifs des files d'assemblages, excités en raison des contacts entre les assemblages et des différences de longueur des files. Ces mouvements correspondent aux modes de fréquence plus élevée, pour lesquels des mouvements de fluide accompagnent les mouvements des assemblages.

La base modale décrite ici a été utilisée pour réaliser des simulations du comportement sismique des cœurs REP. 

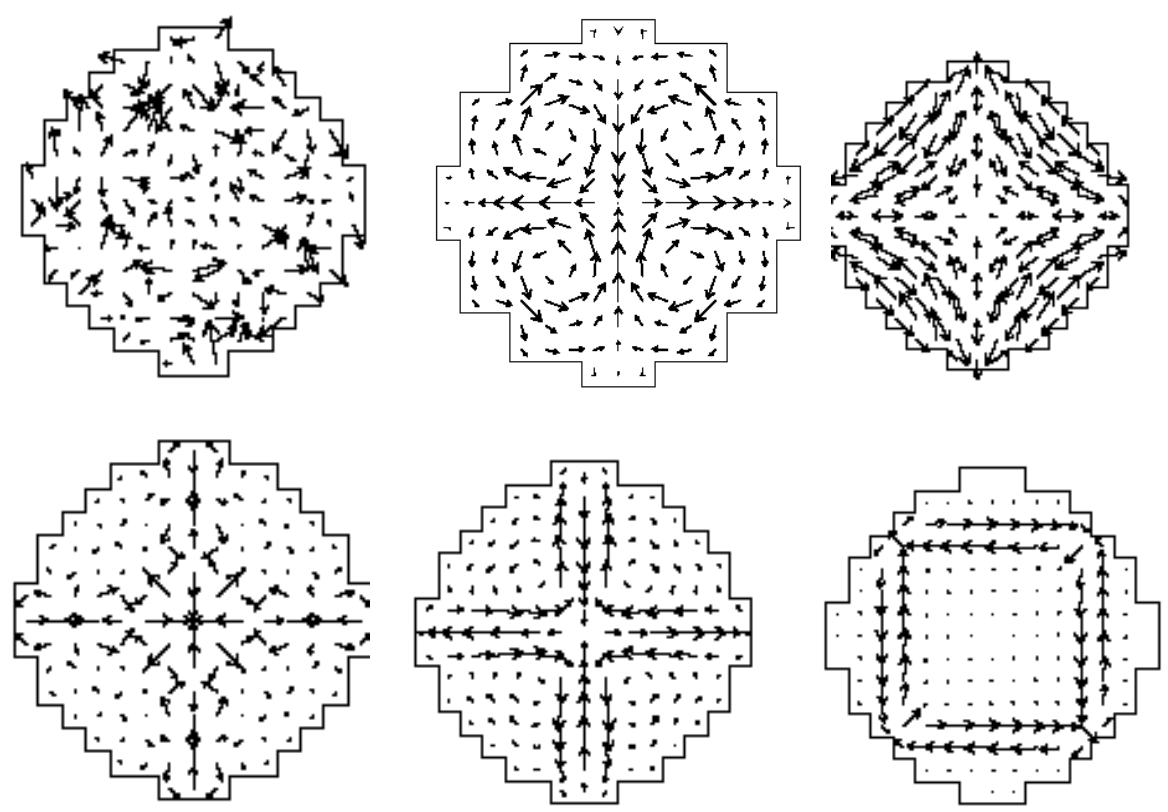

Fig. 5. Modes propres de vibration des assemblages d'un cœur REP.
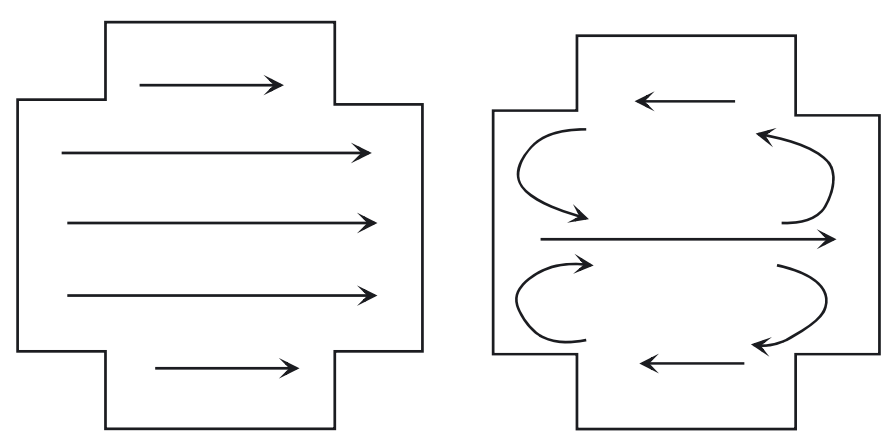

Fig. 6. Types de déplacements des assemblages REP.

\section{2 Étude d'un cas générique : faisceau $10 \times 10$}

Les figures 6-8 présentent des modes propres de vibration d'un faisceau « générique » de $10 \times 10=100$ assemblages, contenu dans une cuve cylindrique (avec un espace d'eau libre). La baisse de fréquence due au fluide est, avec les valeurs numériques utilisées, de $10 \%$ à $15 \%$. La figure 7 présente différents types de modes (déformée et isovaleurs de pression). Le premier mode correspond à des mouvements en opposition de phase des colonnes. Le deuxième mode correspond à un mouvement de « respiration $»$ du faisceau. Pour ces deux modes, il n'y a pas, ou peu, de mouvement global de fluide et les fréquences sont faibles. On constate en particulier que, dans l'espace d'eau libre les pressions sont constantes : il n'y a pas de mouvement fluide.

Les figures 8 et 9 présentent les principaux modes dits « sismiques» correspondant à un mouvement global des assemblages et excités par un séisme. Il y a des mouvements globaux de fluide pour ces modes. La figure 7 présente les modes obtenus « directement », sans homogénéisation et la figure 8 présente les mêmes modes obtenus en utilisant la méthode d'homogénéisation. Il y a des mouvements de fluide et des pressions non constantes dans l'espace de fluide libre. Les deux méthodes fournissent des résultats extrêmement proches. L'analyse de ce cas montre, par comparaison avec le cas des coeurs REP, que la présence d'un espace libre autour du faisceau de tubes modifie fortement l'allure générale des modes propres et donc le comportement vibratoire. Un des enjeux de ces simulations est la validation des méthodes d'homogénéisation : les résultats obtenus par les méthodes d'homogénéisation (avec un coût de calcul plus faible) sont très proches de ceux obtenus en effectuant un calcul complet.

La figure 10 présente un exemple d'application en 3D, avec un réseau de $10 \times 10$ tubes. Des isovaleurs de pression et des déformées des tubes sont présentées.

\section{Conclusion}

Les méthodes d'homogénéisation présentées permettent de réaliser des simulations des phénomènes d'interaction fluide structure pour des faisceaux de tubes immergés dans un fluide. Ces méthodes sont basées sur les équations d'Euler de fluide parfait.

Cette équation ne rend compte, en principe, que des effets inertiels de la présence du fluide. Les effets dissipatifs peuvent cependant être pris en compte sous la forme d'un amortissement forfaitaire de type « amortissement de Rayleigh ».

Une meilleure description de ces effets dissipatifs passe par un saut qualitatif dans les modèles, avec une prise en compte de toute la richesse des écoulements de fluide. 

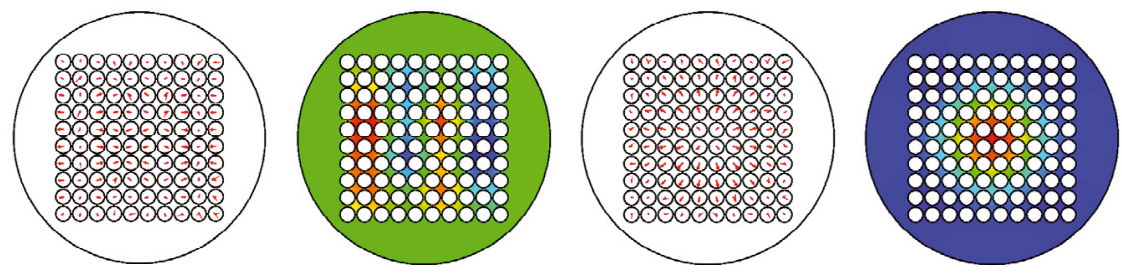

Fig. 7. Modes « direct».
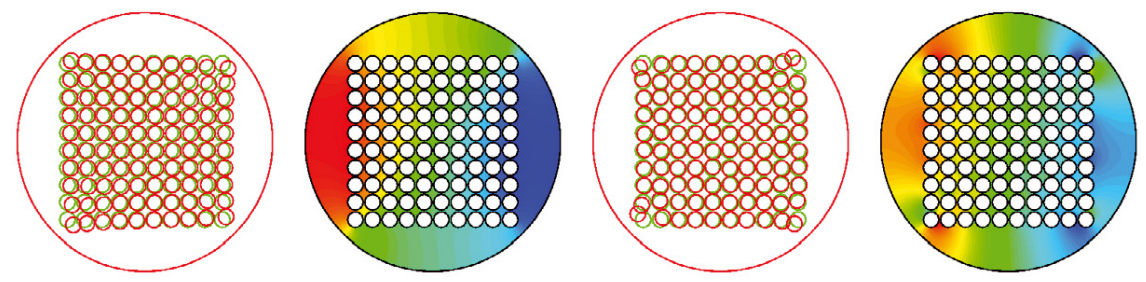

Fig. 8. Modes sismiques « direct $»$.
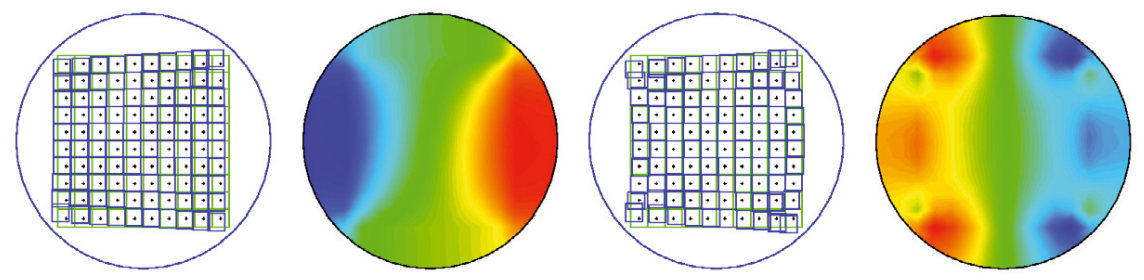

Fig. 9. Modes sismiques homogénéisés.
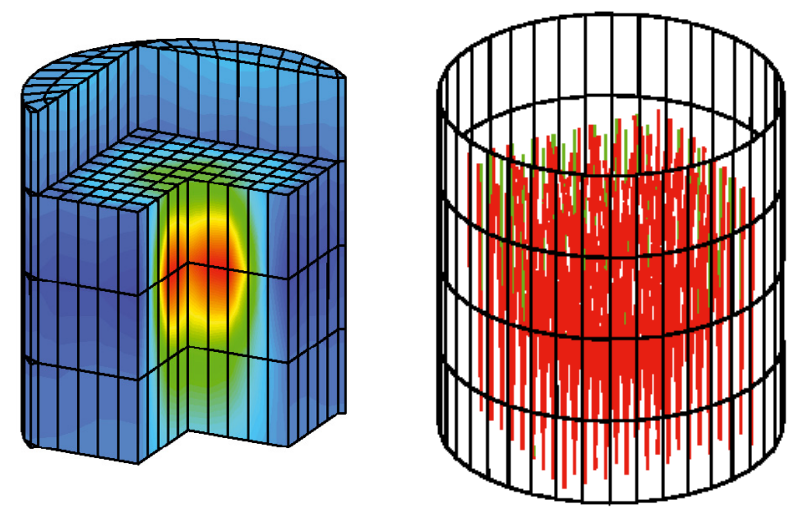

Fig. 10. Cas générique : tridimensionnel.

\section{Références}

[1] E. Delangre, Fluides et solides, Les éditions de l'École Polytechnique, 2001

[2] F. Axisa, Modélisation des systèmes mécaniques, Interactions fluide structure Hermès, 2001

[3] R.-J. Gibert, Vibrations des structures, Collection des études et recherches d'Électricité de France, Eyrolles, 1988
[4] A. Bensoussan, J.L. Lions, G. Papanicolaou, Asymptotic Analysis for Periodic structures, Noerth Holland, 1978

[5] C. Conca, J. Planchard, B. Thomas, M. Vanninathan, Problèmes mathématiques en couplage fluide structure, Collection des études et recherches d'Électricité de France, Eyrolles, 1994

[6] R.J. Zhang, A unified homogenization mode of beam bundle in fluid, J. Press. Vessel Techn. 120 (1998) 56-61

[7] Y. Shinohara, T. Shimogo, Vibrations of square and hexagonal cylinders in a liquid, J. Press. Vessel Techn. 103 (1981) 233-239

[8] D. Bergman et al., Les méthodes d'homogénéisation : théorie et applications en physique, Collection des études et recherches d'Électricité de France, Eyrolles, 1985

[9] L. Hammami, Étude de l'interaction fluide structure dans les faisceaux de tubes par une méthode d'homogénéisation, Application à l'analyse sismique des cœurs RNR, Thèse, Université Paris 6, 1990

[10] K. Cheval, Modélisation du comportement sismique de structures multitubulaires baignées par un fluide dense, Thèse, Université d'Évry, 2000

[11] D. Brochard, F. Gantenbein, R.-J. Gibert, Dynamic analysis of LMFBR core by an homoenization method, ASME PVP, San Diego, 1987

[12] CASTEM : site www.cast3M.fr

[13] J.P. Morand, R. Ohayon, Interactions fluids structures, Masson, 1992 